\title{
LINEAR SERIES WITH AN $N$-FOLD POINT ON A GENERAL CURVE
}

\author{
DAVID SCHUBERT
}

\begin{abstract}
A linear series $(V, \mathscr{L})$ on a curve $X$ has an $N$-fold point along a divisor $D$ of degree $N$ if $\operatorname{dim}\left(V \cap H^{0}(X, \mathscr{L}(-D))\right) \geq \operatorname{dim} V-1$. The dimensions of the families of linear series with an $N$-fold point are determined for general curves.
\end{abstract}

We work over the field of complex numbers $\mathbf{C}$.

Let $X$ be a smooth projective curve. A $g_{d}^{r}$ on $X$ is a linear series of dimension $r$ and degree $d$ on $X$, i.e., a pair $(V, \mathscr{L})$ consisting of a line bundle $\mathscr{L}$ of degree $d$ and an $r+1$ dimensional subspace $V \subset H^{0}(X, \mathscr{L})$. The $g_{d}^{r}$ s on $X$ are parameterized by a projective scheme $G_{d}^{r}(X)$. If $X$ is general in moduli, then $\operatorname{dim} G_{d}^{r}(X)=\rho(g, r, d)=g-(r+1)(g+r-d)$ [ACGH].

Definition. We say that a $g_{d}^{r}(V, \mathscr{L})$ has an $N$-fold point along a divisor $D$ of degree $N \geq 2$ in $X$ if $\operatorname{dim}\left(V \cap H^{0}(X, \mathscr{L}(-D))\right) \geq r$.

If $\pi: X \rightarrow V$ is a flat proper irreducible family of smooth curves, there exists a scheme $G_{d}^{r}(X / V)$ which is projective over $V$ whose fiber over each $v \in V$ is $G_{d}^{r}\left(X_{v}\right)$ where $X_{v}=\pi^{-1}(v)$ [EH-2]. It is easy to see that, by considering the construction of $G_{d}^{r}\left(X \times_{V} X^{N} / X^{N}\right)$ as the degeneracy locus of a vector bundle map, there is a closed subscheme $N(X / V) \subset G_{d}^{r}\left(X \times_{V} X^{N} / X^{N}\right)$ such that the fiber over each point $\left(P_{1}, \ldots, P_{N}\right) \in X_{V}^{N}$ consists of the $g_{d}^{r}$ s on $X_{V}$ with an $N$-fold point along $\sum P_{j}$. We will write $N(X)$ if $V$ is a point.

Let $\mathscr{M}_{g}$ denote the moduli space of smooth curves of genus $g \geq 3$, and let $U$ be the open subset of $\mathscr{M}_{g}$ corresponding to curves without nontrivial automorphisms. Let $Z \rightarrow U$ be the universal curve over $U$. We will prove the following theorem.

Theorem. With $g \geq N \geq 2, g \geq 3, r \geq 2$ and the notation as above, $\operatorname{dim} N\left(Z_{u}\right) \leq \rho(g, r, d)-r(N-1)+N$ when $u$ is a sufficiently general point of $U$.

Received by the editors July 21, 1989.

1980 Mathematics Subject Classification (1985 Revision). Primary $14 \mathrm{H} 10$. 
This extends a result of Marc Coppens who proved the case when $N=2$ [C]. It is easy to see that $\rho(g, r, d)-r(N-1)+N$ is a lower bound for the dimension of $N\left(Z_{u}\right)$ when $N\left(Z_{u}\right) \neq \varnothing$. If $\rho(g, r, d)-r(N-1)+N \geq 0$ and $\rho(g, r-1, d-N) \geq 0$ then $N\left(Z_{u}\right) \neq \varnothing[\mathrm{S}]$.

Let $X$ be a connected complete curve. We say that $X$ is of compact type if and only singularities of $X$ are ordinary double points, and the dual graph is a tree. We say a connected closed subcurve $Y \subset X$ is a tail if it meets $\overline{X-Y}$ at at most one point. We say that a curve $X$ of genus $g$ is of special type if: it is of compact type; each irreducible component is a nonsingular rational or nonsingular elliptic curve; and each irreducible elliptic component is a tail.

We say that a sequence $a=\left(a_{0}, a_{1}, \ldots, a_{r}\right)$ is of type $(r, d)$ if $0 \leq a_{0}<$ $a_{1}<\cdots<a_{r} \leq d$. If $X$ is a smooth curve containing a point $P$, and $L=$ $(V, \mathscr{L})$ is a $g_{d}^{r}$ on $X$, then the orders of vanishing of the sections of $V$ determine a sequence a of type $(r, d)$. We call a the vanishing sequence of $L$ at $P$. We denote by $W(\mathbf{a})=\sum\left(a_{i}-i\right)$ the weight of the sequence a . If $\mathbf{b}=\left(b_{0}, \ldots, b_{r}\right)$ is a sequence of type $(r, d)$, and a $g_{d}^{r} L$ has vanishing sequence $\mathbf{a}=\left(a_{0}, \ldots, a_{r}\right)$ at $P$, we say that $L$ satisfies the vanishing condition b at $P$ if $a_{i} \geq b_{i}$ for $i=0, \ldots, r$.

In $\S 2$ we show the existence of a family of smooth curves $X_{T-\{0\}} \rightarrow T-\{0\}$ which specialize to a curve $X_{0}$ of special type and a family $A^{\prime}$ of $g_{d}^{r}$ s with $N$-fold points on $X_{T-\{0\}} \rightarrow T-\{0\}$. The $N$-fold points of $A^{\prime}$ specialize to a genus $M$ tail of $X_{0}$. The $g_{d}^{r}$ s of $A^{\prime}$ also satisfy a vanishing condition a at a point. The relative dimension of $A^{\prime}$ over $T-\{0\}$ is 0 , and the codimension of $A^{\prime}$ in $N\left(X_{T-\{0\}} / T-\{0\}\right)$ is $\leq N-M+W(\mathbf{a})$.

In $\S 3$ we use the theory of limit linear series as developed by Eisenbud and Harris [EH-2] to show that the crude limit linear series on $X_{0}$ induced by $A^{\prime}$ forces $\rho(g, r, d)-r(N-1)+M-W(\mathbf{a}) \geq 0$.

The products involving $\mathbf{P}^{1}$ in the proof of Lemma 1 are taken over Spec $\mathbf{C}$. All other products are fibered over $\overline{\mathscr{M}}_{g}$ unless specified otherwise.

The author is grateful to Ziv Ran for his generous assistance.

The author would like to thank Marc Coppens for pointing out some errors in the original version of this paper.

We will make use of Knudsen's results concerning stable $n$-pointed curves [K1, K2]. A stable $n$-pointed curve is a connected projective curve $X$ and $n$ distinct nonsingular points $P_{1}, \ldots, P_{n}$ of $X$ such that: the only singularities of $X$ are ordinary double points; and on every smooth rational component $Y \subset X, \#\left\{P_{i} \mid P_{i} \in Y\right\}+\#\{Y \cap \overline{X-Y}\} \geq 3$. For each $g$ and $n$, there exists a coarse moduli space for $n$-pointed stable curves of genus $g$ which we denote by $\overline{\mathscr{M}}_{g, n}$. For each $g$ and $n, \overline{\mathscr{M}}_{g, n}$ is a projective variety.

The functors of relative stable $n$-pointed curves and relative stable $n-1$ pointed curves with an additional section are isomorphic. For each stable $n$ - 
pointed curve $\left(X, P_{1}, \ldots, P_{n}\right)$ there is a curve $X_{c}$ and morphism $c: X \rightarrow X_{c}$ such that: $\left(X_{c}, c\left(P_{1}\right), \ldots, c\left(P_{n-1}\right)\right)$ is a stable $n-1$-pointed curve; and either $c$ is an isomorphism, or $P_{n}$ lies on a rational component $Y \subset X$ whose image in $X_{c}$ is a point and $\left.c\right|_{X-Y}$ is an isomorphism of $X-Y$ with $X_{c}-c(Y)$. When we wish to consider $\overline{\mathscr{M}}_{g, n}$ as coarsely representing the functor of stable $n$-1-pointed curves with an additional section, we will write $\overline{\mathscr{M}}_{g, n} \simeq \bar{Z}_{g, n-1}$. When $n=0$ we will write $\overline{\mathscr{M}}_{g}$ instead of $\overline{\mathscr{M}}_{g, 0}$ and $\bar{Z}_{g}$ instead of $\bar{Z}_{g, 0}$.

For each $m<n$ and each subset $\left\{i_{1}<\cdots<i_{m}\right\}$ of $\{1, \ldots, n\}$, we have a contraction morphism $\pi: \overline{\mathscr{M}}_{g, n} \rightarrow \overline{\mathscr{M}}_{g, m}$ obtained by forgetting the points not indexed by $\left\{i_{1}<\cdots<i_{m}\right\}$ and collapsing certain rational subcurves, if necessary.

There is a natural clutching morphism

$$
\gamma: \overline{\mathscr{M}}_{g_{1}, n+1} \times_{\mathbf{C}} \overline{\mathscr{M}}_{g_{2}, m+1} \rightarrow \overline{\mathscr{M}}_{g_{1}+g_{2}, n+m} .
$$

If $\left(X, P_{1}, \ldots, P_{n+1}\right)$ and $\left(Y, Q_{1}, \ldots, Q_{m+1}\right)$ correspond to a point $(x, y) \in$ $\overline{\mathscr{M}}_{g_{1}, n+1} \times_{\mathbf{C}} \overline{\mathscr{M}}_{g_{2}, m+1}$, then $\gamma(x, y)$ corresponds to the $n+m$-pointed curve obtained by joining $X$ and $Y$ at the points $P_{n+1}$ and $Q_{m+1}$.

Fix $g \geq 3,2 \leq N \leq g, r \geq 2$, and $d$. As before, we let $U \subset \overline{\mathscr{M}}_{g}$ be the open subset corresponding to smooth curves without nontrivial automorphisms, and we let $\pi: Z \rightarrow U$ be the universal curve over $U$. Let $H \subset N(Z / U)$ be a component of the scheme of $g_{d}^{r}$ 's with $N$-fold points whose dimension is maximal with respect to the property that $\pi(H)=U$.

We have a natural morphism $\alpha: H \rightarrow\left(\bar{Z}_{g}\right)^{N}$. Let $B$ be the closure of $\alpha(H)$ in $\left(\bar{Z}_{g}\right)^{N}$, and let $\beta: B \rightarrow \overline{\mathscr{M}}_{g}$ be the natural morphism. Note that $\beta(B)=\overline{\mathscr{M}}_{g}$, because $\pi(H)=U$. Let $M=N-\min \left\{\operatorname{dim} \beta^{-1}(x) \mid x \in \overline{\mathscr{M}}_{g}\right\}$.

Note that $0 \leq M \leq N$. If $M=0$, then the theorem holds, because the $g_{d}^{r}$ s on a general curve with an $N$-fold point along a divisor whose support is a general point have codimension $r(N-1)$ by Theorem 4.5 of [EH-2]. We will henceforth assume $M \geq 1$.

Lemma 1. There exists a point $b \in B$ corresponding to a curve $X_{b}$ which is of special type and points $P_{1}, \ldots, P_{N} \in X_{b}$ which lie on a tail of genus $\leq M$ or $P_{1}=P_{2}=\cdots=P_{N}$ is a nonsingular point on a rational component of $X_{b}$.

Proof. All products involving $\mathbf{P}^{1}$ in this proof are fibered over Spec $\mathbf{C}$. All other products are fibered over $\mathscr{M}_{g}$.

There is a set-theoretic map on closed points $\delta: \overline{\mathscr{M}}_{0, g} \times\left(\bar{Z}_{g}\right)^{N} \rightarrow\left(\bar{Z}_{0, g}\right)^{N}$ which we will now describe. A point $w \in \overline{\mathscr{M}}_{0, g} \times\left(\bar{Z}_{g}\right)^{N}$ corresponds to a $g$-pointed rational curve $\left(X, Q_{1}, \ldots, Q_{g}\right)$ and $N$ points $P_{1}, \ldots, P_{N}$ on the stable genus $g$ curve $\tilde{X}_{Q_{1}, \ldots, Q_{g}}$ obtained by attaching a fixed elliptic curve $E$ to $X$ at each point $Q_{1}, \ldots, Q_{g}$. Let $\eta: \widetilde{X}_{Q_{1}, \ldots, Q_{g}} \rightarrow X$ be the natural map which collapses the elliptic tails. The point $\delta(w) \in\left(\bar{Z}_{0, g}\right)^{N}$ corresponds to $\left(X, Q_{1}, \ldots, Q_{g}\right)$ and the $N$ points $\eta\left(P_{1}\right), \ldots, \eta\left(P_{N}\right)$. 
For each $I=\left(i_{1}, \ldots, i_{N}\right)$ such that $0 \leq i_{j} \leq g$ for $j=1, \ldots, N$ there is a scheme $D_{I}$ which parameterizes $g$-pointed rational curves $\left(X, Q_{1}, \ldots, Q_{g}\right)$ and points $P_{1}, \ldots, P_{N}$ on $\widetilde{X}_{Q_{1}, \ldots, Q_{g}}$ such that each $P_{j}$ lies on the elliptic tail attached to $X$ at the point $Q_{i_{j}}$ if $i_{j}>0$, or $P_{i_{j}}$ lies on $X$ if $i_{j}=0$. Each $D_{I}$ is easily shown to exist by considering contraction and clutching morphisms.

Furthermore, there are morphisms $\phi_{I}: D_{I} \rightarrow\left(\bar{Z}_{0, g}\right)^{N}$ and $\Psi_{I}: D_{I} \rightarrow \overline{\mathscr{M}}_{0, g} \times$ $\left(\bar{Z}_{g}\right)^{N}$ such that $\delta \circ \Psi_{I}=\phi_{I}$. It follows that $\delta\left(p_{Z}^{-1}(B)\right)$ is a closed subset of $\left(\bar{Z}_{0, g}\right)^{N}$ where $p_{Z}$ is the projection of $\overline{\mathscr{M}}_{0, g} \times\left(\bar{Z}_{g}\right)^{N}$ to $\left(\bar{Z}_{g}\right)^{N}$.

There are morphisms

$$
\zeta_{N}:\left(\bar{Z}_{0, g}\right)^{N} \rightarrow\left(\bar{Z}_{0,3}\right)^{N} \simeq\left(\mathbf{P}^{1}\right)^{N} \text { and } \zeta_{g}:\left(\bar{Z}_{0, g}\right)^{g} \rightarrow\left(\bar{Z}_{0,3}\right)^{g} \simeq\left(\mathbf{P}^{1}\right)^{g}
$$

which correspond to forgetting about the last $g-3$ points on a $g$-pointed rational curve and designating the first three points as 0,1 and $\propto$. The morphisms $\zeta_{N}$ and $\zeta_{g}$ are products of contraction morphisms. There is a morphism $\varepsilon: \overline{\mathscr{M}}_{0, g} \rightarrow\left(\bar{Z}_{0, g}\right)^{g}$ which corresponds to associating the $g$-pointed rational curve $\left(X, Q_{1}, \ldots, Q_{g}\right)$ to itself and the points $Q_{1}, \ldots, Q_{g}$ on $X$. The morphism $\varepsilon$ exists because of the functorial properties $\overline{\mathscr{M}}_{0, g}$ and $\bar{Z}_{0, g}$. We get a set-theoretic map

$$
f=\left(\zeta_{g} \circ \varepsilon \circ p_{M}\right) \times\left(\zeta_{N} \circ \delta\right): \overline{\mathscr{M}}_{0, g} \times\left(\bar{Z}_{g}\right)^{N} \rightarrow\left(\mathbf{P}^{1}\right)^{g} \times\left(\mathbf{P}^{1}\right)^{N}
$$

where $p_{M}$ is the projection of $\overline{\mathscr{M}}_{0, g} \times\left(\bar{Z}_{g}\right)^{N}$ onto $\overline{\mathscr{M}}_{0, g}$. Furthermore, $f\left(p_{Z}^{-1}(B)\right)$ is closed in $\left(\mathbf{P}^{1}\right)^{g} \times\left(\mathbf{P}^{1}\right)^{N}$.

We will prove the lemma by showing that there exists a point

$$
\left(Q_{1}, \ldots, Q_{g}, P_{1}, \ldots, P_{N}\right) \in f\left(p_{Z}^{-1}(B)\right) \subset\left(\mathbf{P}^{1}\right)^{g} \times\left(\mathbf{P}^{1}\right)^{N}
$$

such that either: (i) $P_{1}=\cdots=P_{N}$ and at most $M$ of the $Q_{i}$ are equal to $P_{1}$; or (ii) there exists a point $Q \in \mathbf{P}^{1}$ such that all of the $P_{i}$ are distinct from $Q$ and at least $g-M$ of the $Q_{i}$ are equal to $Q$. Suppose that $\left(X, Q_{1}, \ldots, Q_{g}\right)$ is a stable $g$-pointed rational curve. Let $\widetilde{X}_{Q_{1}, \ldots, Q_{g}}$ be the curve obtained by attaching the elliptic curve $E$ at each $Q_{i}$, and let $\eta: \widetilde{X}_{Q_{1}, \ldots, Q_{g}} \rightarrow X$ be the map which collapses the elliptic tails as before. The map $\bar{Z}_{0, g} \rightarrow \bar{Z}_{0,3} \simeq \mathbf{P}^{1}$ corresponding to forgetting about the last $g-3$ points of a $g$-pointed rational curve and designating the first three points as 0,1 and $\propto$ induces a morphism $X \rightarrow \mathbf{P}^{1}$ and hence a morphism $\widetilde{X}_{Q_{1}, \ldots, Q_{g}} \rightarrow \mathbf{P}^{1}$. Note that if $P$ is a point in $\mathbf{P}^{1}$ and $k$ of the points $Q_{1}, \ldots, Q_{g}$ map to $P$, then the pre-image of $P$ in $\tilde{X}_{Q_{1}, \ldots, Q_{g}}$ is a tail of genus $k$. hence condition (i) above implies that there exists a point in $B$ corresponding to a curve and $N$ points which lie on a tail of genus $\leq M$. Note that the preimage of $\mathbf{P}^{1}-\{P\}$ in $\widetilde{X}_{Q_{1}, \ldots, Q_{g}}$ is a tail of genus $g-k$. Thus condition (ii) will also imply the lemma. 
Let $D_{k}=\left\{\left(P_{1}, \ldots, P_{N}\right) \in\left(\mathbf{P}^{1}\right)^{N} \mid\right.$ at least $k$ points coincide $\}$. We will use the fact that if $Y$ is a closed subset of $\left(\mathbf{P}^{1}\right)^{N}$ and $\operatorname{dim}(Y) \geq k-1$, then $D_{k} \cap Y \neq \varnothing$. This fact follows from: the diagonal is ample in $\mathbf{P}^{1} \times \mathbf{P}^{1}$; thus $\operatorname{dim}\left(D_{k} \cap Y\right) \geq 1$ implies $\operatorname{dim}\left(D_{k+1} \cap Y\right) \neq \varnothing ;$ and $\operatorname{codim} D_{k}=k-1$.

Let $p_{g}$ and $p_{N}$ denote the projections of $\left(\mathbf{P}^{1}\right)^{g} \times\left(\mathbf{P}^{1}\right)^{N}$ to $\left(\mathbf{P}^{1}\right)^{g}$ and $\left(\mathbf{P}^{1}\right)^{N}$, respectively.

The dimension of the fiber of $f\left(p_{Z}^{-1}(B)\right)$ over each point of $(0,1, \propto) \times$ $\left(\mathbf{P}^{1}\right)^{g-3}$ is $\geq N-M$, because the dimension of the fiber of $B$ over each point of $\overline{\mathscr{M}}_{g}$ is $\geq N-M$. Hence

$$
p_{g}\left(f\left(p_{Z}^{-1}(B)\right) \cap\left(\mathbf{P}^{1}\right)^{g} \times D_{N-M+1}\right)=(0,1, \infty) \times\left(\mathbf{P}^{1}\right)^{g-3} .
$$

Let $\kappa=\max \left\{k \mid\left(\mathbf{P}^{1}\right)^{g} \times D_{k} \cap f\left(p_{Z}^{-1}(B)\right) \neq \varnothing\right\}$. We will prove the lemma by showing that condition (i) holds if $\kappa=N$ and condition (ii) holds if $\kappa \leq N-1$.

Suppose $\kappa=N$. Then $p_{g}\left(f\left(p_{Z}^{-1}(B)\right) \cap\left(\mathbf{P}^{1}\right)^{g} \times D_{N}\right)$ has codimension $\leq$ $N-(N-M+1)=M-1$ in $(0,1, \infty) \times\left(\mathbf{P}^{1}\right)^{g-3}$. Hence

$$
\operatorname{dim}\left(p_{g}\left(f\left(p_{Z}^{-1}(B)\right) \cap\left(\mathbf{P}^{1}\right)^{g} \times D_{N}\right)\right) \geq g-2-M .
$$

Let $E_{M+1}=\left\{\left(Q_{1}, \ldots, Q_{g}\right) \in(0,1, \infty) \times\left(\mathbf{P}^{1}\right)^{g-3} \mid\right.$ at least $M+1$ of the points $Q_{1}, \ldots, Q_{g}$ coincide $\}$. We have

$$
\operatorname{dim}\left(E_{M+1}\right)=g-3-M<\operatorname{dim} p_{g}\left(f\left(p_{Z}^{-1}(B)\right) \cap\left(\mathbf{P}^{1}\right)^{g} \times D_{N}\right) .
$$

Thus we can find $\left(Q_{1}, \ldots, Q_{g}, P_{1}, \ldots, P_{N}\right) \in f\left(P_{Z}^{-1}(B)\right)$ so that $P_{1}=\cdots=$ $P_{N}$ and at most $M$ of the $Q_{i}$ 's are equal to $P_{1}$.

Suppose $\kappa \leq N-1$. Let $W$ be a component of $f\left(p_{Z}^{-1}(B)\right) \cap\left(\mathbf{P}^{1}\right)^{g} \times D_{\kappa}$. Then $p_{N}(W)$ is a point $\left(P_{1}, \ldots, P_{N}\right) \in\left(\mathbf{P}^{1}\right)^{N}$, because otherwise $\left(\mathbf{P}^{1}\right)^{g} \times$ $D_{\kappa+1} \cap f\left(P_{Z}^{-1}(B)\right) \neq \varnothing$. Choose a puint $R \in \mathbf{P}^{1}$ so that $R \neq P_{i}$ for $i=$ $1, \ldots, N$. If $\kappa=N-1$, we choose $R \in\{0,1, \infty\}$. Now $W$ has codimension $\leq \kappa-(N-M+1)$ in $f\left(p_{Z}^{-1}(B)\right) \cap\left(\mathbf{P}^{1}\right)^{g} \times D_{N-M+1}$ so $p_{g}(W)$ has codimension $\leq \kappa-(N-M+1)$ in $(0,1, \infty) \times\left(\mathbf{P}^{1}\right)^{g-3}$. Thus $\operatorname{dim} p_{g}(W) \geq g-M$ if $\kappa \leq N-2$, and $\operatorname{dim} p_{g}(W) \geq g-M-1$ if $\kappa=N-1$. Since $p_{g}(W)$ is closed in $(0,1, \propto) \times\left(\mathbf{P}^{1}\right)^{g-3}$ there are $\operatorname{dim}\left(p_{g}(W)\right)$ factors of $\left(\mathbf{P}^{1}\right)^{g-3}$ so that the projection of $p_{g}(W)$ to the product of these factors is onto. Thus there is a point $\left(Q_{1}, \ldots, Q_{g}\right) \in p_{g}(W) \subset(0,1, \propto) \times\left(\mathbf{P}^{1}\right)^{g-3}$ so that at least $g-M$ of the $Q_{i}$ 's are equal to $R$. Thus condition (ii) holds and the lemma follows.

We can find a smooth curve $T$ containing a point 0 and a morphism $\phi: T \rightarrow$ $B \subset\left(\bar{Z}_{g}\right)^{N}$ such that $\phi(0)$ is the point $b \in B$ described in Lemma 1 , and the induced map $T \rightarrow \overline{\mathscr{M}}_{g}$ sends $T-\{0\}$ to the subset of $U$ where the fibers of $\beta: B \rightarrow \overline{\mathscr{M}}_{g}$ have dimension $N-M$.

After replacing $T$ with a base extension, if necessary, there is a family of 0-pointed stable curves $X \rightarrow T$ which corresponds to the morphism $T \rightarrow \overline{\mathscr{M}}_{g}$. 
Note that if $T$ is replaced with a base extension and the singularities of $X$ are resolved by blowing up, the curve $X_{0}$ will change by inserting chains of rational curves at the nodes of $X_{0}$. Thus we may assume (by replacing $T$ with a base extension and blowing up the singularities of $X$ if necessary) that there is a family of curves $X \rightarrow T$ which extends $Z \times_{U}(T-\{0\})$ and has the following properties: (1) $X_{0}$ is of special type; (2) there is a tail $Y$ of $X_{0}$ of genus $\leq M$ so that the sections $s_{i}: T \rightarrow X$ induces by the map $\phi: T \rightarrow B \subset\left(\bar{Z}_{g}\right)^{N}$ are such that each $s_{i}(0)$ is smooth point of $X_{0}$ which lies in $Y$ (if $P_{1}=P_{2}=\cdots=P_{N}$ is a point on a rational component of $X_{b}$ then, after blowing up, there will exist $s_{i}$ such that the $s_{i}(0)$ lie on a tail of genus 0 in $\left.X_{0}\right) ;(3)$ there is a section $s: T \rightarrow X$ such that $s(0)$ is a smooth point of $X_{0}$ which lies in a rational component of $X_{0}-Y$; and (4) $X$ is smooth. Note that these properties are unchanged if $T$ is replaced with a base extension which sends one point to 0 and the singularities of the new $X$ are resolved by blowing up.

Theorem 2. There is a sequence a of type $(r, d)$ and a closed subscheme $A$ of $H \times_{B}(T-\{0\})$ such that: $A$ consists of all linear series in $H \times_{B}(T-\{0\})$ which satisfy vanishing condition a along $s(T-\{0\})$; the fibers $A_{t}$ are nonempty for each $t \in T-\{0\} ;$ and $\operatorname{dim} A_{t}=0$ for all but finitely many $t \in T-\{0\}$.

Proof. Note that $H \times_{B}(T-\{0\})$ is proper over $T-\{0\}$, and the subset of linear series satisfying a particular vanishing condition along $s(T-\{0\})$ is closed. Since there are only finitely many sequences of type $(r, d)$, the lemma is a consequence of the following.

Lemma 2a. Let $X$ be a smooth curve. Let $A$ be a closed subset of $G_{d}^{r}(X)$, and let a be a sequence of type $(r, d)$. If $\operatorname{dim} A \geq 1$ and every linear series in $A$ satisfies vanishing condition a at a point $P \in X$, then there exists a sequence $\mathbf{a}^{\prime}$ with $a_{i}^{\prime} \geq a_{i}$ for $i=0, \ldots, r$ and $a_{k}^{\prime}>a_{k}$ for some $k$ such that $A$ contains a linear series which satisfies vanishing condition $\mathbf{a}^{\prime}$ at $P$.

Proof. We may assume $A$ is irreducible. Consider the natural map $\Phi: A \rightarrow$ $\operatorname{Pic}^{d}(X)$. Suppose for some $x \in \operatorname{Pic}^{d}(X)$, the fiber $\Phi^{-1}(x)$ has dimension $\geq 1$, and let $\mathscr{L}$ be the line bundle corresponding to $x$. The set of $(r+1)$ dimensional vector spaces of $H^{0}(X, \mathscr{L})$ which have vanishing sequence a at $P$ is a Schubert variety, and hence is affine. It follows that $A$ contains a $g_{d}^{r}$ which does not have vanishing sequence a at $P$, because $\Phi^{-1}(x)$ is closed and $\operatorname{dim} \Phi^{-1}(x) \geq 1$. So we may assume $\Phi(A)$ contains a closed curve in $\operatorname{Pic}^{d}(x)$.

If the lemma were false, then there would exist a family $F$ of $g_{d-a_{r}}^{0}$ 's obtained from $A$ by taking $\left(H^{0}\left(Y, \mathscr{L}\left(-a_{r} P\right)\right) \cap V, \mathscr{L}\left(-a_{r} P\right)\right)$ for every $(V, \mathscr{L})$ of $A$. But a family of $g_{d-a_{r}}^{0}$ 's is a family of divisors of degree $d-a_{r}$. Since $\operatorname{dim}(F) \geq 1$, it must contain a divisor with $P$ in its support. But the linear series in $A$ associated with this divisor satisfies vanishing condition $\left(a_{0}, \ldots, a_{r-1}, a_{r}+1\right)$. 
If $T$ is replaced with an appropriate base extension, and the singularities of $X$ blown up, we may also assume that $A \rightarrow T-\{0\}$ gives an isomorphism $A^{\prime} \rightarrow T-\{0\}$ for some component $A^{\prime}$ of $A$.

The codimension of $H \times_{B} T$ in $H \times_{U} T$ is $N-M$, and $A^{\prime}$ has codimension $\leq W(\mathbf{a})$ in $H \times_{B} T$. Thus the theorem will follow when we show that $W(\mathbf{a})+$ $N-M \leq \rho(g, r, d)-r(N-1)+N$.

The family of curves $X \rightarrow T$ and $A^{\prime}$ determine a crude limit linear series on $X_{0}$ [EH-2]. This crude limit linear series is a collection consisting of a $g_{d}^{r}$ for each of the components of $X_{0}$. If $C$ is a component of $X_{0}$, the $g_{d}^{r}\left(V_{C}, \mathscr{L}_{C}\right)$ on $C$ is determined in the following manner. Let $R$ be the local ring of $T$ at 0 , and let $\eta$ be the generic point of $\operatorname{Spec} R$. Then $A^{\prime}$ determines a unique line bundle $\mathscr{L}^{C}$ on $X_{\text {Spec } R}$ such that the restriction of $\mathscr{L}^{C}$ to $X_{0}$ has degree 0 on every component of $X_{0}$ except $C$. Also, $A^{\prime}$ determines a subspace $V \subset H^{0}\left(X_{\eta},\left.\mathscr{L}^{C}\right|_{X_{\eta}}\right)$. Let $\tilde{V}_{C}=V \cap H^{0}\left(X_{\operatorname{Spec} R}, \mathscr{L}^{C}\right)$. Then $V_{C}=$ $\widetilde{V}_{C} \otimes k(0) \subset H^{0}\left(X_{0},\left.\mathscr{L}^{C}\right|_{X_{0}}\right)$ and $\mathscr{L}_{C}=\left.\mathscr{L}^{C}\right|_{C}$ where $k(0)$ the residue field of $R$.

Let $Q=Y \cap\left(\overline{X_{0}-Y}\right)$ and let $Y^{\prime}$ be the component of $Y$ containing $Q$. Let $Y_{1}, \ldots, Y_{k}$ be the remaining components of $Y$. In addition to parameterizing a family of $g_{d}^{r}$ 's on $X_{T-\{0\}} \rightarrow T-\{0\}, A^{\prime}$ also determines a family of $g_{d-N}^{r-1}$ 's corresponding to the sections which vanish along $\sum s_{i}(T-\{0\})$.

Let $\left(W_{Y^{\prime}}, \eta_{Y^{\prime}}\right)$ be the $g_{d-N}^{r-1}$ on $Y^{\prime}$ of the limit $g_{d-N}^{r-1}$ on $X_{0}$. Then

$$
\eta^{Y^{\prime}}=\mathscr{L}^{Y^{\prime}}\left(-\sum s_{i}(T)-\sum n_{i} Y_{i}\right) \quad \text { where } n_{i} \geq 0
$$

so $\eta_{Y^{\prime}}=\left.\eta^{Y^{\prime}}\right|_{Y^{\prime}}=\mathscr{L}_{Y^{\prime}}\left(-\sum Q_{i}\right)$ where the $Q_{i}$ are points not equal to $Q$. Also,

$$
W_{Y^{\prime}}=\left(\tilde{V}_{Y^{\prime}} \cap H^{0}\left(X_{\text {Spec } R^{\prime}} \eta^{Y^{\prime}}\right)\right) \otimes k(0) \subset V_{Y^{\prime}} \cap H^{0}\left(\eta_{Y^{\prime}}\right) \text {. }
$$

So the vanishing sequence b of $\left(W_{Y^{\prime}}, \eta_{Y^{\prime}}\right)$ at $Q$ is a subsequence of the vanishing sequence $\mathrm{c}$ of $\left(V_{Y^{\prime}}, \mathscr{L}_{Y^{\prime}}\right)$ at $Q$.

The following lemma is an immediate consequence of Theorem 4.5 of [EH-2] and Theorem 2.3 of [EH-1].

Lemma 3. If $C$ is a genus $g$ curve of special type and $P$ and $Q$ are two smooth points in rational components of $C$, then the existence of a crude limit $g_{d}^{r}$ on $C$, which satisfies vanishing conditions a and b at points $P$ and $Q$, respectively, implies that $\rho(g, r, d)-W(\mathbf{a})-W(\mathbf{b}) \geq 0$.

Now the crude limit $g_{d-N}^{r-1}$ on $X_{0}$ determined by $A^{\prime}$ restricts to a limit $g_{d-N}^{r-1}$ on the genus $M$ tail $Y$ which satisfies vanishing condition b at $Q$. Hence $\rho(M, r-1, d-N)-W(\mathbf{b}) \geq 0$. Since $\mathbf{b}$ is a subsequence of $\mathbf{c}$ we have $W(\mathbf{c}) \leq W(\mathbf{b})+d-r$. So $W(\mathbf{c}) \leq \rho(M, r-1, d-N)+d-r$. 
Let $F$ be the component of $\overline{X_{0}-Y}$ which contains $Q$. If $\mathbf{c}^{\prime}$ is the vanishing sequence of $V_{F}$ at $Q$, then the definition of crude limit linear series requires

$$
\begin{aligned}
W\left(c^{\prime}\right) & \geq(r+1)(d-r)-W(c) \\
& \geq r(d-r)-\rho(M, r-1, d-N) .
\end{aligned}
$$

Now the crude limit $g_{d}^{r}$ on $X_{0}$ determined by $A^{\prime}$ restricts to a crude limit $g_{d}^{r}$ on $\overline{X-Y}$ satisfying vanishing conditions $\mathrm{c}$ at $Q$ and $\mathbf{a}$ at $s(0)$. Thus,

$$
\begin{aligned}
0 \leq & \rho(g-M, r, d)-W(\mathbf{a})-W\left(\mathbf{c}^{\prime}\right) \\
\leq & \rho(g-M, r, d)-W(\mathbf{a})+\rho(M, r-1, d-N)-r(d-r) \\
= & (r+1)(d-r)-r(g-M)-w(\mathbf{a}) \\
& +r(d-N-r+1)-(r-1) M-r(d-r) \\
= & (r+1)(d-r)-r g-W(\mathbf{a})-r(N-1)+N-(N-M) \\
= & \rho(g, r, d)-W(\mathbf{a})-r(N-1)+N-(N-M) .
\end{aligned}
$$

Thus $W(\mathbf{a})+N-M \leq \rho(g, r, d)-r(N-1)+N$, and the theorem follows.

\section{REFERENCES}

[ACGH] E. Arbarello, M. Cornalba, P. Griffiths, and J. Harris, Geometry of algebraic curves, Vol. 1, Springer, New York, 1984.

[C] M. Coppens, A remark on the embedding theorem for general smooth curves, Preprint No. 405, Univ. of Utrecht, 1986.

[EH-1] D. Eisenbud and J. Harris, Divisors on general curves and cusptial rational curves, Invent. Math. 74 (1983), 371-418.

[EH-2] _ Limit linear series: basic theory, Invent. Math. 85 (1986), 337-371.

[K1] F. Knudsen, The projectivity of the moduli space of stable curves. II: The stacks $M_{g, n}$, Math. Scand. 52 (1983), 161-199.

[K2] _ The projectivity of the moduli space of stable curves. III: The line bundles on $M_{g, n}$, and a proof of the projectivity of $\bar{M}_{g, n}$ in characteristic 0, Math. Scand. 52 (1983), 200212.

[S] D. Schubert, Linear series with cusps and n-fold points, Trans. Amer. Math. Soc. 304 (1987), 689-703.

Department of Mathematics, State University of New York, College at Geneseo, Geneseo, New York 14454 